\title{
Microbiota-mediated protection against antibiotic-resistant pathogens
}

\author{
Rekha B. Panwar ${ }^{1} \cdot$ Richard P. Sequeira $^{1}$ Thomas B. Clarke $\mathbb{1}^{1}$ \\ Received: 4 December 2020 / Revised: 19 March 2021 / Accepted: 9 April 2021 / Published online: 4 May 2021 \\ (c) The Author(s) 2021. This article is published with open access
}

\begin{abstract}
Colonization by the microbiota provides one of our most effective barriers against infection by pathogenic microbes. The microbiota protects against infection by priming immune defenses, by metabolic exclusion of pathogens from their preferred niches, and through direct antimicrobial antagonism. Disruption of the microbiota, especially by antibiotics, is a major risk factor for bacterial pathogen colonization. Restoration of the microbiota through microbiota transplantation has been shown to be an effective way to reduce pathogen burden in the intestine but comes with a number of drawbacks, including the possibility of transferring other pathogens into the host, lack of standardization, and potential disruption to host metabolism. More refined methods to exploit the power of the microbiota would allow us to utilize its protective power without the drawbacks of fecal microbiota transplantation. To achieve this requires detailed understanding of which members of the microbiota protect against specific pathogens and the mechanistic basis for their effects. In this review, we will discuss the clinical and experimental evidence that has begun to reveal which members of the microbiota protect against some of the most troublesome antibioticresistant pathogens: Klebsiella pneumoniae, vancomycin-resistant enterococci, and Clostridioides difficile.
\end{abstract}

\section{Introduction-microbiota development and importance}

In humans, and other mammals, all surfaces exposed to the environment are home to myriad archaea, bacteria, viruses, and eukaryotic microbes $[1,2]$. These colonizing microbes are referred to as the microbiota. For their hosts they provide a variety of benefits, including the provision of nutrients, protection against infections, and maturation of the immune system [2, 3]. In exchange these organisms are provided with a nutrient-rich habitat [1]. The principal system we have for interacting with the microbiota is the immune system [4-7]. The central role of these microbes in programming our immune system, and host health more

These authors contributed equally: Rekha B. Panwar, Richard P. Sequeira

Thomas B. Clarke

Thomas.Clarke@imperial.ac.uk

1 MRC Centre for Molecular Bacteriology and Infection, Department of Infectious Disease, Imperial College London, London, UK broadly, has been demonstrated in both human and animal studies, where microbiota disruption or depletion has been linked to a range of diseases and immune dysfunctions, including autoimmunities [8-10] and increased susceptibility to infection by all classes of pathogens [4, 5, 11-17]. Better understanding how host and microbiota interact may therefore allow us to minimize disruption to the microbiota to prevent disease, and also harness the power of the microbiota to treat disease.

The microbiota develops in an ordered manner with transitions that mirror many of the major steps in host development [18-21]. Prior to birth, host mucosal and skin epithelia are not thought to be colonized by live microbial communities, then, immediately after birth, the process of microbiota colonization begins [22, 23]. In newborns all skin and mucosal epithelia have a similar microbiota composition and it is thought that this initial seeding microbiota is derived from the mothers' vaginal and intestinal microbiota [2, 23-25]. By contrast in babies born by caesarian section the microbiota of the newborn does not reflect the mothers' vaginal microbiota but more closely resembles her skin microbiota for the first few months of life [18, 26, 27]. This highlights how swift the acquisition of the microbiota is during the birthing process and how amenable the mucosa of newborns is to microbial colonization. Over the next 
2-3 years the microbiota of each different mucosal site develops and differentiates such that each mucosal site has its own unique microbiota [19]. The balance of factors that shape the microbiota is thought to reflect the environmental, nutritional, microbial, and immunological challenges at each site. In the intestine, for example, the process of microbiota development is strongly influenced by host diet, with the lactobacilli that are part of the seeding inoculum continuing to dominate until weaning where the transition to solid foods corresponds to the development of more anaerobic bacterial communities from the Bacteroidia and Clostridia taxa $[1,17,28,29]$. In the upper respiratory tract, the process of differentiation can occur quickly, beginning after only a week of life but the factors driving this are less clear $[30,31]$. In parallel to microbiota development the development of immune system occurs. Many aspects of the production and functional maturation of the immune cells are shaped by the acquisition of the microbiota in tissues throughout the body [4, 5, 32-34]. The parallel development of the microbiota and immune system suggests a reciprocal relationship whereby not only does the microbiota drive immune development but also that the immune system can shape and control the microbiota [35]. The orchestrated patterns of immune and microbiota development that occurs in mammals result in the establishment of a highly effective barrier against infectious disease in adulthood $[3,5,36]$. Host resistance to infection is therefore established by the microbiota either through its direct antimicrobial activity or indirectly through its regulation of the immune system, particularly the innate immune system.

\section{The microbiota as a regulator of innate immune development and function}

The innate immune system is the first arm of the immune system to respond to microbial infection [37]. Historically, the production and functional maturation of the cells that constitute this branch of the immune system have been thought of as controlled by host signals alone [37-39]. This view has been increasingly revised as our understanding of the role played by the microbiota in mammalian physiology increases $[6,40]$. It is now clear that many aspects of innate immune cells have their function modified by the microbiota. The epithelium is the hosts' first tissue to interact with the microbiota, it must therefore act as a barrier to prevent invasion of the microbiota into deeper, more sensitive host tissues. Its role is not simply limited to acting as physical barrier to colonizing microbes but it is also a central component of host defenses through its own antimicrobial activity and its communication with underlying immune tissues [41]. The microbiota influences many of the functions of the epithelium [42]. From the prospective of barrier function development, the acquisition of the microbiota drives the production of mucus and stimulates epithelial production of certain antimicrobial peptides (AMP) [21, 43]. Within the intestine, mucus thickness reflects bacterial load, with the mucus layers in the colon being the thickest as this site has the highest bacterial burden along the gastrointestinal length [44]. Tolerance to the microbiota to limit pathological inflammation is thought to develop through a number of mechanisms. In mice, restriction of epithelial innate pattern recognition receptor activation driven by the microbiota, and in particular Toll-like receptor signaling, occurs via proteasomal degradation and microRNA-mediated inhibition of downstream signaling cascade components [45]. In human intestinal epithelial cells, reduced TLR expression [46] and negative regulation of NF- $\mathrm{KB}$ activity by commensal microbes have been demonstrated [47-49] and this is proposed to help mediate tolerance to the microbiota. The epithelium is therefore constantly responding to microbial cues and its function is attuned to establish symbiosis with the microbiota.

Neutrophils are essential for protection against infection and nearly every aspect of the neutrophil life-cycle is now thought to be influenced by signals from the microbiota $[5,6]$. The development of the microbiota from its neonatal to adult composition stimulates the production of neutrophils in the bone marrow [15]. This occurs through microbiota-mediated stimulation of intestinal IL-17A that leads to increased levels of circulating granulocyte colony stimulating factor that promotes neutrophil production [15]. Then, upon release of these neutrophils into the circulation, the influence of the microbiota continues. In the absence of the microbiota, the ability of circulating neutrophils to enter host tissues in response to inflammation is diminished, as is the bactericidal activity of these cells $[14,50]$. In addition, in the absence of cues from the microbiota, the rate of neutrophil apoptosis is accelerated [51], underlining the comprehensive influence that the microbiota has on neutrophil biology and the ability of these cells to control microbial infection.

Macrophages and dendritic cells, often collectively referred to as mononuclear phagocytes, form a critical collection of cells that are central to host defenses to infection [52, 53]. Macrophages are found in tissues throughout the body. In addition to their role in host defense, they maintain tissue homeostasis and repair damage in injured tissues. Macrophage phenotype and function is exquisitely attuned to the physiology of their host tissue. For example, in the intestine, macrophages have dampened responses to microbial stimulation that reflects the need to tolerate the enormous microbial load in the intestine [54], and in the lung alveolar, macrophages are required to prevent the accumulation of pulmonary surfactants needed for correct lung function [55]. The instructions 
that ensure that tissue macrophage function is correctly aligned with host tissue biology are not solely derived from the host. Within the intestine the number and function of macrophages is shaped by the microbiota. The preponderance of intestinal macrophages are derived from circulating monocytes and this process of replacement is driven by the microbiota through CCR2 activity [56]. Functionally, microbiota-derived short-chain fatty acids (SCFA) dampen the inflammatory response of intestinal macrophages while enhancing their antimicrobial activity $[57,58]$. Similarly, the microbiota also primes the antimicrobial activity of intestinal macrophages through induction of cytokines such as IL-36 to control pathogen levels in the intestine [17]. Likewise, in the lung, the antimicrobial activity of alveolar macrophages is enhanced by the presence of the microbiota but the number of macrophages there, and in other extra-intestinal tissues, seems to be somewhat less influenced by the microbiota $[13,59,60]$. The function of dendritic cells in different host tissues is similarly influenced by the microbiota. Within the intestine a variety of microbiota-derived molecules have been found to influence the function of dendritic cells. Like macrophages, SCFA have been shown to reduce inflammatory cytokine production by dendritic cells thus promoting the induction of regulatory $\mathrm{T}$ cells (Tregs) [61]. Likewise, one of the capsular polysaccharides produced by the commensal Bacteroides fragilis (PSA) drives the production of the antiinflammatory cytokine IL-10 that in turn leads to the induction of Tregs [62]. The microbiota also exerts influence on dendritic cells outside of the intestine. In the absence of the microbiota, during respiratory infection by influenza the migration of dendritic cell from the lung to the mediastinal lymph nodes is reduced, and this is associated with reduced T-cell responses and reduced anti-influenza antibody responses [63]. In addition, the microbiota regulates the activity of lung dendritic cells to make them better able to induce IgA class-switching in B cells resulting in better responses to intranasal vaccines [64]. Dendritic cells in non-mucosal tissues also have their function impacted by the microbiota. Cytokine production, and in particular interferon production, in splenic dendritic cells is enhanced by the microbiota and this promotes natural killer cellmediated protection against systemic viral infection [59]. As these examples demonstrate, the microbiota, and in particular the intestinal microbiota, has a significant impact on the development and functional programming of innate immunity in tissues throughout the body.

The inflammatory set-point established by the microbiota is therefore important for the elimination of pathogens and prevention of infection. Equally important, however, is the regulation of immunity to prevent overt responses that can lead to loss of tolerance to commensals, inflammation, autoimmunity, or increased risk of infection and cancer [65]. Maintenance of homeostasis is therefore critical within the gastrointestinal tract where the greatest abundance of microbes engage with host immune cells. Members of the lymphocyte lineage regulate much of this control over the immune system. In generating proinflammatory responses, Th17 cells are important producers of IL-17 that activate epithelial cells to produce AMP, enhance immune responses, and recruit neutrophils to control infections [66-69]. The microbiota have also been shown to be important in the development of these $\mathrm{T}$ cells. Segmented filamentous bacteria have demonstrated the ability to induce Th17 accumulation by promoting IL-1 $\beta$ and IL-23 production from phagocytes [69, 70]. Similarly, ATP from the microbiota promotes Th17 differentiation [70]. An important counterpart to Th17 cells are Tregs that are an essential regulator of immunity through the production of anti-inflammatory mediators such as IL-10 $[71,72]$. Members of the microbiota have also been shown to support the development of Tregs. Commensal Clostridia, in particular clusters IV and XIVa, enriched TGF- $\beta$ and accumulation of Treg in the colon [73]. This intimate balance between an inflammatory and regulatory immune response driven by these T-cell populations is underscored by the fact that Tregs, Th17 cells, and innate lymphoid cells (ILC) 3 all share a common nuclear receptor (retinoic acid-related orphan receptor $\gamma \mathrm{t}$ ) and that differentiation requires a balance of signals to determine the functional fate [74]. Understanding this balance between immune responses driven by these lymphocytes is complex but is thought to play a critical role in the development of diseases such ulcerative colitis and Crohn's disease where imbalance of immune responses to microbiota, diet, and self-antigens leads to chronic inflammation and damage. ILCs are critical cells that coordinate the activity of innate and adaptive immune cells within the gut through their production of these important signaling molecules. While the complexity of ILC functions in the gut is not fully understood, there is some evidence that the microbiota is important for regulating their functions. ILC 3 limit effector T-cell responses to the microbiota, as well as being a major source of IL-22 within the intestine. Thus, their loss results in dysregulated adaptive immune responses and inflammation toward commensals [75-77]. Epithelial production of IL-25 in response to the microbiota helps dampen the inflammatory products of ILC3 and IgA production promoted by ILC2 is regulated by the microbiota [78, 79]. Sensing of microbial signatures by macrophages leads to their production of IL-1 $\beta$ that induces ILC 3 to feedback to mononuclear phagocytes instructing IL-10 production and subsequent expansion of Tregs promoting greater immune tolerance [80]. The microbiota is therefore constantly interacting with innate and adaptive immune cells in the intestine and this controls the inflammatory tone and the 
set-point of intestinal antibacterial defenses. Harnessing the protective power of the intestinal microbiota therefore represents an exciting opportunity to treat infection. The intestinal microbiota is also, however, a reservoir of many pathogens that go on to cause acute infection [81]. Below, we outline the delicate balance that exists in the intestine between the beneficial microbiota and some of the most problematic antibiotic-resistant pathogens for human health.

\section{The intestinal microbiota and antibiotic- resistant pathogens}

For many bacterial pathogens, including Klebsiella pneumoniae, vancomycin-resistant enterococci (VRE), and Clostridioides difficile, colonization of the intestinal tract is the first step on the pathway to acute infection [3, 36, 81, 82]. Despite these three pathogens ultimately causing a different spectrum of diseases, colonization of the intestinal tract is a shared point in their pathogenesis [36, 81]; thus, understanding this key step in disease could lead to treatments that are broadly effective against multiple pathogens.

Klebsiella pneumoniae is a Gram-negative Proteobacteria belonging to the family Enterobacteriaceae. It is commensal of the gastrointestinal and upper respiratory tract in several mammals, including humans, with human carriage estimated to be around 3-8\% [83, 84]. In hospital patients, the incidence of gastrointestinal colonization can be significantly higher (up to $38 \%$ ) [85]. Beyond its human host, it is also found in environmental reservoirs such as water, sewage, and soil [86]. In a fraction of its hosts, $K$. pneumoniae causes respiratory, urinary tract, wound, and bloodstream infections [87, 88]. Classically, it has been considered an opportunistic nosocomial pathogen with higher incidence in neonates, the elderly, immunocompromised, and notably patients receiving antibiotic treatment [84]. This view of $K$. pneumoniae as a purely nosocomial pathogen has, however, been reevaluated with a growing number of infections now also being identified in the community and not solely in patients with comorbidities [89]. K. pneumoniae capable of infecting these mostly healthy patients in a community setting is thought to be more virulent, and is associated with specific capsule types [90]. The development of antibiotic resistance has also amplified the importance of $K$. pneumoniae infections. Following the first European case of an extended-spectrum $\beta$-lactamase in 1983 , carbapenems became vital in treating these resistant organisms [91]. However, resistance to these last-line defense antibiotics has expanded where carbapenem-resistant $K$. pneumoniae (CR-Kp) clinical isolates rose from $1.6 \%$ in 2001 to $10.4 \%$ in 2011 [91]. Similar rates of CR-Kp have increased in China from 2.4 to $13.4 \%$ between 2005 and 2016. The Centers for Disease Control and Prevention have thus declared the emergence of these carbapenem-resistant Enterobacteriaceae an urgent threat to public health owing to the lack of treatment options and high fatality rate. Gastrointestinal colonization is the pivotal step in K. pneumoniae pathogenesis [85]. Studies examining $K$. pneumoniae carriage and subsequent infection have attempted to identify factors that predispose a patient to colonization [92]. Duration of hospital exposure and usage of antibiotics increase the rate of $K$. pneumoniae colonization [92]. Length of stay is likely to increase the probability of a patient being exposed to the bacteria via healthcare workers and fomites. Antibiotic usage causes dysbiosis of the microbiota providing favorable conditions for $K$. pneumoniae [17, 93, 94]. Limited data are available on which antibiotics predispose to K. pneumoniae colonization but some clinical studies have demonstrated a correlation between vancomycin treatment and subsequent $K$. pneumoniae colonization $[93,94]$. Supporting this idea vancomycin addition to a simulated intestinal microbial system allowed the expansion of $K$. pneumoniae [95]. The utility of the microbiota for preventing $K$. pneumoniae colonization is supported by studies where fecal microbiota transplant has been shown to promote the clearance of $K$. pneumoniae from the intestine in both humans and animal models $[17,96,97]$. Studies using animal models have shown that of the major bacterial phyla in the intestinal microbiota, it is members of the Bacteroidetes, but not the Firmicutes, Proteobacteria, or Actinobacteria, that protect against $K$. pneumoniae colonization [17]. Bacteroidetes provide protection via the stimulation of the intestinal immune system. Specifically, the development of Bacteroidetes post weaning primes the production of intestinal IL-36 $\gamma$ that stimulates a macrophage-dependent barrier against $K$. pneumoniae [17]. This priming of intestinal IL-36 requires that Bacteroidetes intimately associate with the intestinal mucosal and this is dependent on the Bacteroidetes commensal colonization factors that promote the association of these organisms with the mucosa [17]. Other studies demonstrate that protection against $K$. pneumoniae by the microbiota is not limited to indirect stimulation of the immune system but can also occur via direct inhibition from the microbiota. Specifically, production of SCFA by the microbiota leads to intracellular acidification of $K$. pneumoniae thus inhibiting its growth [98]. Within the other major human niche of $K$. pneumoniae, the upper airway, the microbiota is seemingly less able to prevent colonization. Again, in animal models, after direct inoculation of $K$. pneumoniae into the upper airway $K$. pneumoniae is able to successfully compete with the upper airway microbiota and establish colonization [17]. Successful colonization of the upper airway requires production of the capsular polysaccharide [17]. Capsular polysaccharide is a 
well-established virulence factor important for evading innate immune defenses during acute infection [88]. There is a multitude of different capsule types produced by different strains of $K$. pneumoniae. Capsular composition has a designated $\mathrm{K}$ nomenclature based on the $\mathrm{K}$-antigen, of which there has been thought to be a total of 77 different types [99]. Originally done using agglutination, the advancement of technologies for whole genome sequencing suggests there may be even more than 138 distinct $\mathrm{K}$ types [100]. Importantly, the vast majority of the infections in the community are from hypervirulent strains of the serotype $\mathrm{K} 1$ and $\mathrm{K} 2$ capsule types only [101-103]. The capsule may support initiating gastrointestinal colonization but is seemingly unimportant for persistent intestinal colonization, unlike its established role in colonization in the urinary tract or its contribution to immune evasion [104-108]. In the absence of the capsule, $K$. pneumoniae can no longer compete with the upper airway microbiota and is cleared from this site [17]. Clearance is driven by the stimulation of innate immunity by commensal Proteobacteria in the upper airway, rather than Bacteroidetes, and via IL-17A activity, rather than IL-36 [17]. If $K$. pneumoniae does manage to evade the intestinal microbiota and immune defenses that inhibit its colonization, it can go on to cause lung infection. Nevertheless, the intestinal microbiota is still important in resisting disease in this distal tissue. Members of the intestinal, and upper airway, microbiota that are potent stimulators of the pattern recognition receptor Nod2 enhance early innate clearance of $K$. pneumoniae from the lung by alveolar macrophages through a IL-17A, GM-CSF, and ERK-dependent pathway [13]. This highlights the complexity of the interaction between the microbiota, immune system, and $K$. pneumoniae. At different mucosal sites there are different commensal groups that are having different effects on the immune system that may, or may not, lead to resistance to colonization and thus infection. Enterococci are part of the human microbiota but two members of this genus are significant human pathogens: E. faecalis and E. faecium [109-111]. Enterococci are the major Grampositive coccus in the human intestinal microbiota and commensal strains can be present at levels of up to $10^{7}$ $\mathrm{CFU} / \mathrm{g}$ in feces $[110,111]$. These organisms are naturally resistant to many antibiotics and can easily acquire further resistant elements leading to many strains being multidrug resistant. Disease-causing enterococci are responsible for systemic infections, urinary tract infections, and endocarditis $[109,112]$. A feature of enterococcal biology is that strains associated with disease are often markedly different to commensal enterococci. Commensal strains have smaller genomes and disease-causing strains having a broader repertoire of carbohydrate utilization pathways allowing use of host-derived glycans [110, 113, 114]. This may underlie why commensal strains rarely cause disease. It has been known for a number of years that antibiotic therapy facilitates the expansion of VRE in the intestine $[115,116]$. Of particular note is that human and animal studies have found that the suppression of VRE is particularly associated with anaerobic bacteria indicating that these commensals are particularly important in keeping the levels of enterococci in check $[116,117]$. There have been a number of pathways delineated by which the microbiota provides protection against VRE expansion. Protection has been shown to be via both the stimulation of the immune system and also through the direct antagonism of commensals against VRE. Microbiota-mediated resistance to intestinal VRE colonization driven by the immune system can occur via the stimulation of the innate immune system. During homeostasis the microbiota provides basal stimulation to the innate immune system through agonism of pattern recognition receptors, fortifying intestinal antimicrobial defenses [43]. In the intestine, the microbiota activates Toll-like receptors on stromal cells that stimulate the production of the AMP REG3 $\gamma$ [43], indicating that while the innate immune system in the intestine has its activity restrained to prevent pathological inflammation its responsiveness is not eliminated. Simple restoration of a pure TLR agonist was sufficient to stimulate this immune defense pathway and protect against VRE [43]. There are further more indirect immunological defenses that restrict enterococcal expansion through the activity of the IL-22 receptor. Signaling through the IL-22 receptor has been proposed to limit the levels of intestinal enterococci by promoting the fucosylation of host glycans [118]. It is thought that these act as a nutrient source for intestinal anaerobes, such as Bacteroides, promoting their expansion thereby limiting enterococcal numbers [118]. Further studies have begun to delineate at lower taxonomic levels specific members of the microbiota that protect against VRE. By comparing mouse microbiota that provide different levels of protection against VRE colonization, and then isolating commensals from the protective microbiota by culturing, a simple consortium of four commensals was identified that protect against VRE [11]. This consortium consisted of Clostridium bolteae, Blautia producta, Bacteroides sartorii, and Parabacteroides distasonis. Within this consortium it is Blautia producta that is directly antagonistic to VRE through the elaboration of a lantibiotic that is inhibitory to VRE [119]. Similarly, in patients, the enrichment of lantibiotic genes was associated with reduced E. faecium being detected in their stool [119]. Enterococci are also thought to be directly antagonistic to one another through the production of bacteriocins. E. faecalis strains that produce bacteriocins from the pPD1 plasmid are able to outcompete non-bacteriocin producing strains in the intestine, including VRE [120].

Of all infections associated with the disruption of the microbiota, it is Clostridioides difficile infection that is 
perhaps the most well known and clinically the most common [121, 122]. C. difficile is an obligate, Grampositive anaerobe that exists in two states: a vegetative state, which is the metabolically active form of $C$. difficile; and the spore form, which is the dormant state used by $C$. difficile to survive harsh environmental conditions often during host-to-host transmission [123]. C. difficile causes a range of diseases from asymptomatic intestinal colonization through to fulminant colitis [122, 123]. Again, like the other pathogens discussed in this review, animal models and human studies have demonstrated that both the microbiota and immune system are important in controlling $C$. difficile infection. While most antibiotics that disrupt the microbiota can increase susceptibility to $C$. difficile, it is thought that fluoroquinolones, clindamycin, and cephalosporins are the major antibiotics that render patients susceptible to C. difficile [124, 125]. Diet composition has also been shown to be important in microbiota-mediated protection against $C$. difficile infections. Excess zinc has been shown to increase susceptibility and severity of $C$. difficile infections by altering the microbiota and reducing the effectiveness of calprotectin reduces zinc availability to the pathogen [126]. Increasing dietary carbohydrates has been shown to affect the microbiota leading to greater SCFA production that is inhibitory to $C$. difficile [127, 128]. A diet rich in carbohydrates instead of protein selects for organisms in the microbiota, such as the Lachnospiraceae, that are able to metabolize these carbohydrates but also the rarer amino acids that limit the availability of amino acids that C. difficile requires [129, 130]. A case study has supported how dietary intervention with a specific carbohydrate prevented $C$. difficile reinfection in patients [131]. C. difficile has two major virulence factors that are critical determinants of acute disease; these are toxin A and toxin B [132]. These proteins can inactivate rho GTPases that in intestinal epithelial cells causes cell death and thus ultimately barrier disruption [132]. Signaling through Toll-like receptor and Nod-like receptors, Myd88, and the cytokines IL-22 and IL17A has been shown to be required for immune protection against $C$. difficile [123, 133-136]. In addition to innate immunity in the stromal cells of the intestine, resistance to C. difficile requires ILC, specifically ILC1, and multiple granulocytes (neutrophils and eosinophils) [137, 138]. One of the major risk factors for $C$. difficile infection is prior antibiotic therapy [122]. Initial treatment of infection often relies on the antibiotics metronidazole and vancomycin [122, 139]; however, recurrence of infection can occur in up to $35 \%$ of patients and has been associated with immunosuppression and further antibiotic therapy [140, 141]. While still not generally used as an initial treatment for $C$. difficile, fecal microbiota transplantation is used to treat recurrent C. difficile infection and it is perhaps the best practical demonstration of the power and utility of microbiota-based therapeutics to treat human disease, with success rates of up to $90 \%$ [142, 143]. There are, however, a number of potential drawbacks including related to the uncharacterized nature of the fecal material used for transplantation that could result in the transfer of potential pathogens or the implantation of a microbiota that has deleterious effects on host metabolism [144]. Because of the limitations of using fecal material there have been efforts to understand whether there are specific members of the microbiota that are the key protective commensals preventing $C$. difficile infection, and also to understand the mechanisms by which the microbiota prevents $C$. difficile infection that could be a more refined way to protect against $C$. difficile. Mechanistically, there have been a number of stages in the process of $C$. difficile pathogenesis that the microbiota has been shown to be inhibitory. First is through commensals out competing C. difficile for nutrients. After antibiotic treatment, depletion of commensals that normally consume host-derived sialic acids and succinate limiting their availability leads to an outgrowth of $C$. difficile because of the ready availability of these molecules as a nutrient source [145]. Expression of genes involved in host $N$-linked glycosylation has been demonstrated to be regulated by IL-22. Such glycosylation supports the growth of Phascolarctobacterium that are efficient at utilizing these host molecules and luminal succinate thereby diminishing their nutritional availability to C. difficile [146]. Second, the microbiota protects against $C$. difficile through the modification of another set of hostderived molecules, bile acids. Bile acids are a family of molecules that facilitate fat digestion and absorption, and within the intestine these molecules can undergo chemical transformation by members of the microbiota [147]. Bile acids unmodified by commensal bacteria are referred to as primary bile acids, whereas those that have undergone processing by commensals are referred to as secondary bile acids [147]. The relationship between bile acids and $C$. difficile is complex. Spore germination is thought to be promoted by cholic acids, whereas chenodeoxycholic acids are inhibitory to spore germination [148]. Taurocholic acid is deconjugated by bile salt hydrolases produced by commensals and it has been demonstrated using mouse models whose intestinal conditions are permissive to $C$. difficile growth due to antibiotic treatment that can be reversed by treatment with bile salt hydrolase producing bacteria [149]. This likely reduces the levels of taurocholic acid therefore reducing the germination signal for $C$. difficile limiting its expansion. Another study has shown that modification of primary bile acids by the activity of dehydroxylating enzymes also prevents $C$. difficile infection by inhibiting growth [150]. This work identified a key member of the microbiota important for this dehydroxylation step, Clostridium scindens, which was associated with protection against $C$. difficile in humans and was effective at 
preventing $C$. difficile infection in mice [150]. Similarly, other murine studies have found that protection against C. difficile does not require a complete, diverse microbiota and that a simple consortium of six commensals could protect against $C$. difficile infection [151]. This limited commensal consortia approach is supported by a Danish study in humans where patients were given a consortium of 10-12 commensals spanning the major human intestinal microbiota phyla (including three members of the Bacteroidetes, two members of the Proteobacteria, and seven members of the Firmicutes) that were shown to be effective at curing $C$. difficile in approximately $64 \%$ of patients [152]. This, again, highlights the utility of the microbiota in protecting against $C$. difficile and establishes the feasibility of simple, defined commensal consortia to protect against disease. The mechanistic basis by which this simple consortium is protective in humans is currently unknown. If this were deduced it could allow redesign of the consortium to attempt to increase the cure rate.

\section{Conclusion}

The examples outlined above demonstrate that the microbiota protects against numerous major human bacterial pathogens at multiple stages in their pathogenesis (Fig. 1). The microbiota is therefore an important and highly effective component of host defenses against infection. It is, however, a component of host defenses that is exquisitely sensitive to disruption. A common thread throughout the work described above is that even a transient disruption to the microbiota can eliminate some of its protective effects. This is perhaps most evident during, and even post, antibiotic treatment that can lead to pathogen colonization and expansion, especially in the intestine. The presence of the microbiota makes colonization almost impossible for many bacterial pathogens but these same pathogens can reach levels many orders of magnitude greater after only a single dose of antibiotics that disrupt the microbiota. This bacterial bloom in the gut can have devastating consequences for the host, as data now support that it is often the expansion of these colonizing pathogens that proceeds more serious acute infections [85, 153]. Understanding how bacterial pathogens can so rapidly capitalize of these defects in microbiotamediated defenses may therefore provide unexplored avenues of investigation to develop new ways to prevent infections by these organisms. Despite their obvious importance and utility, another consistent theme emerging from many studies of these pathogen is that antibiotic overuse can cause multiple problems for patients. First is the well-characterized selection for resistant strains of $K$. pneumoniae, VRE, and $C$. difficile and second is the removal of the protective barrier formed by the microbiota.
This only emphasizes further need for more judicious use of antibiotics and the development of alternative approaches, such as using microbiota-based therapeutics to combat bacterial pathogens. A further theme apparent from the mechanistic studies of microbiota-mediated protection against different pathogens is that for a given pathogen the microbiota provides a number of layers of host defense. For example, for $K$. pneumoniae, the intestinal microbiota protects against intestinal colonization by $K$. pneumoniae through direct antibacterial antagonism [98] and through the enhancement of IL-36-mediated defenses [17]. Furthermore, if $K$. pneumoniae go on to cause acute lung infection, signals from the intestinal microbiota enhance respiratory innate immunity too [13]. Thus, multiple aspects of Klebsiella pathogenesis are controlled by the microbiota that could be important for the design of protective commensal consortia. It would be advantageous to design consortia where each member provides this multilayered protection. For example, Bacteroidetes that are also potent activators of the Nod-like receptors would provide protection against both intestinal colonization and pneumonia.

To fully utilize the microbiota to protect against infections there are a number of areas that need to be understood in greater detail. As outlined above, there are an increasing number of preclinical studies that have identified microbiota members that militate against infectious disease; however, one of the biggest hurdles that currently prevent these organisms being pursued further is the difficulties in engrafting them into a new microbiota [154]. This problem is similar to the process of antibiotic drug discovery whereby promising candidate small molecules identified to be inhibitory in vitro fail because of their poor pharmacological properties in vivo. The ability of a microbiota to resist the entry of new microbial members, colonization resistance, is therefore not limited to resistance to pathogens and seemingly extends to myriad other non-pathogenic microbes including those able to form commensal relationships with mammals. A recent study of human colonization by a number of popular probiotics found that there was only patchy colonization of the host after probiotic consumption [154]. To get around this issue for the microbiota means that the rules of colonization need to be better understood. How are these microbial communities assembled and maintained, and how can we use this information to circumvent the colonization resistance encountered by potentially protective commensals? It has been shown that the ability of probiotics to establish themselves at the intestinal mucosa inversely correlates with the levels of these species in the microbiota before probiotic administration [154]. This could suggest that there is some form of niche exclusion occurring, analogous to that of enterococci outlined above [120], that this is at least partly responsible for colonization resistance encountered by 


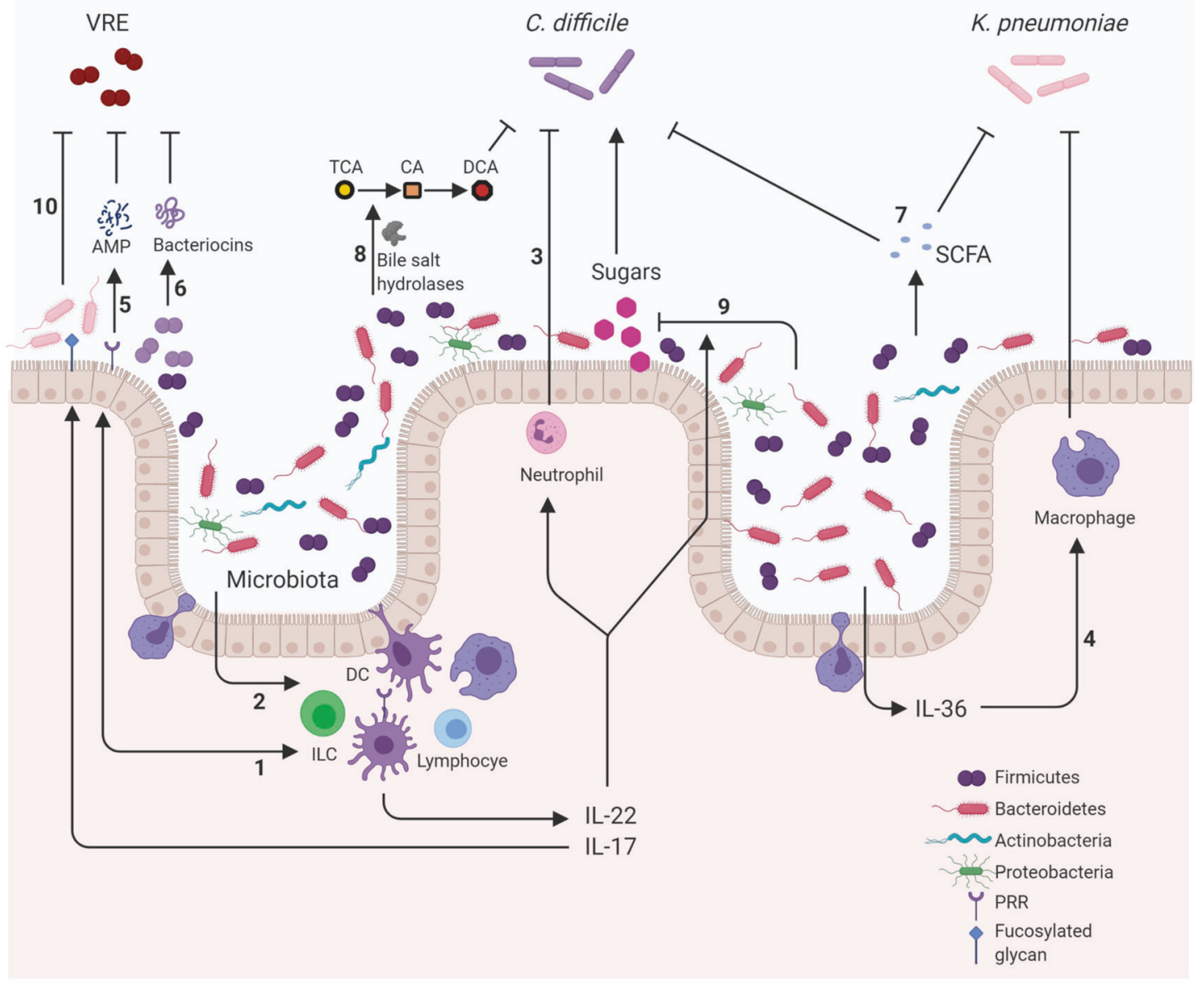

Fig. 1 Mechanisms of microbiota-mediated colonization resistance against pathogens within the gastrointestinal tract. Representation of pathways involving components of the immune system and microbiota that help suppress gastrointestinal tract colonization by Clostridioides difficile, Klebsiella pneumoniae, and vancomycinresistant enterococci (VRE). Suppressive mechanisms involve a variety of important pathways that can be broadly classified into four interconnected categories. Cellular interaction: microbiota activating intestinal epithelia (1) or immune cells (2) induce production of proinflammatory cytokines such as IL-22 and IL-17. These cytokines promote clearance of $C$. difficile through the regulation of important innate cells such as neutrophils (3). Important phyla such as Bacteroidetes induce the production of IL-36 that promotes macrophagemediated clearing of $K$. pneumoniae (4). Antimicrobial production: pattern recognition receptor (PRR) detection of the microbiota induces the production of antimicrobial peptides (AMP) such as REGIII $\gamma$ that is protective against VRE (5). Members of the microbiota can also produce their own AMPS such as bacteriocins. For example, Blautia producta produce lantibiotics that inhibit VRE (6). Metabolic:

commensals. As discussed above, microbiota disruption by antibiotics is one of the main drivers of pathogen colonization and expansion. This could be used as an opportunity by providing a window for the introduction of protective species during or after the completion of antibiotics, thus exploiting microbiota disruption to implant protective metabolic products from the microbiota, including short-chain fatty acids (SCFA), can be antagonistic to pathogens reducing the fitness of C. difficile and acidifying K. pneumoniae intracellularly (7). Enzymes produced by the microbiota can also metabolize host compounds into products that are disruptive to pathogens. The bile acid taurocholic acid (TCA) is deconjugated by microbiota bile acid hydrolases into cholic acid (CA) and subsequently into deoxycholic acid (DCA) that is inhibitory to $C$. difficile growth (8). Nutritional immunity: nutrients are limited resources and so utilization by the microbiota diminishes availability to incoming pathogens. IL-22-induced $\mathrm{N}$-glycosylation promotes microbiota that utilize sialic acid and succinate reducing their abundance preventing the expansion of $C$. difficile (9). Similarly, IL-22 induction of glycan fucosylation promotes anaerobic commensals competing with VRE limiting its expansion (10). These examples demonstrate how the microbiota provide resistance to three pathogens by engaging with a multitude of mechanisms that are antagonistic to the success of the pathogens in the gastrointestinal tract. DC dendritic cell, ILC innate lymphoid cell. Created with BioRender.com.

commensals rather than letting this niche be filled by pathogenic organisms. A further issue is that the range of probiotics that are considered safe for human consumption is small, in comparison to the spectrum of microbial symbionts in the intestine [155]. The available repertoire of protective microbes is therefore limited and likely does not 
cover the entire range of taxa that may be important in protecting against pathogenic microbes. Increasing the pool of suitable organisms is therefore required, but this is not straightforward as it has to be ensured that they will not cause infections themselves, they will not spread antibiotic resistance, do not produce toxins, and there is no genetic drift during their propagation from parental stocks. This suggests that further consideration as to which is the best method to harness the protective effects of the microbiota therapeutically is necessary. Should we use live bacteria, bacterial components, or promote the growth of protective organisms through dietary modification? Each of these approaches comes with their own advantages and disadvantages. The upside of using live bacteria is that, if they successfully colonize the host, they can be self-maintaining and thus can provide longer-term protection compared with using components of these protective organisms that may only have a transient influence on the host because they are more rapidly excreted and are not self-perpetuating. As just described, live bacteria have a number of potential downsides therefore bacterial components or metabolites are an alternative to live bacteria, and include molecules like SCFA. The defined nature of these molecules means that dosing can be more precise and preparations of these molecules can be more consistent. There remains, however, only a limited number of molecules identified from the microbiota where their mode and full spectrum of activity is completely defined [156]. SCFA are some of the most well-characterized microbiota-derived molecules but they influence myriad cells and this pervasive activity needs to be carefully considered before they can be deployed to ameliorate diseases associated with microbiota disruption [157]. A nice example of a strategy utilizing a microbial product was observed in the attempt to deliver the bacteriocin thuricin $\mathrm{CD}$ to attenuate $C$. difficile. It was demonstrated that rectal delivery of thuricin $C D$ induced clearance of $C$. difficile from the colon of mice [158]. Dietary modification could be used to promote the outgrowth of beneficial or protective microbiota members and circumvents the difficulties encountered by the use of whole bacteria or bacterial components. This approach, however, is highly dependent on the initial conditions of the microbiota. Given the complexity of the microbiota, even after disruption by antibiotics, it is hard to predict whether a given dietary modification will have the desired effect because the relationship between diet and microbiota composition is still poorly understood in detail [159]. For example, even if dietary modifications do reconfigure the microbiota, it is unclear how long these take and if this timing varies between people too widely it might render this approach not clinically useful. A further alternative is to bypass the microbiota completely and focus on the immune pathways the microbiota regulates. For example, cytokines like IL-22 and IL-36 are induced by the microbiota and protect against infection, targeting these pathways directly to enhance immunological defenses might be an effective and controlled way to combat infections. Much more needs to be understood about signaling molecules like IL-22 and IL-36, however, before this can be pursued further. For example, will this type of approach work in immunocompromised patients and how will the protective effects against infection be balanced against the potential overt inflammatory response these molecules can induce? These difficulties should not distract from the vast potential of the microbiota as an alternative means to combat infection by antibiotic-resistant pathogens. This potential will be most rapidly realized through increased mechanistic understanding of the interplay between the microbiota, immune system, and pathogenic microbes. As our understanding of how the microbiota inhibits bacterial infections increases so will our ability to use the microbiota to treat antibiotic-resistant infection.

Acknowledgements TBC is a Sir Henry Dale Fellow jointly funded by the Wellcome Trust and Royal Society (Grant Number 107660/Z/ 15Z). RBP is funded by a studentship from Nestle.

\section{Compliance with ethical standards}

Conflict of interest The authors declare no competing interests.

Publisher's note Springer Nature remains neutral with regard to jurisdictional claims in published maps and institutional affiliations.

Open Access This article is licensed under a Creative Commons Attribution 4.0 International License, which permits use, sharing, adaptation, distribution and reproduction in any medium or format, as long as you give appropriate credit to the original author(s) and the source, provide a link to the Creative Commons license, and indicate if changes were made. The images or other third party material in this article are included in the article's Creative Commons license, unless indicated otherwise in a credit line to the material. If material is not included in the article's Creative Commons license and your intended use is not permitted by statutory regulation or exceeds the permitted use, you will need to obtain permission directly from the copyright holder. To view a copy of this license, visit http://creativecommons. org/licenses/by/4.0/.

\section{References}

1. Ley RE, Peterson DA, Gordon JI. Ecological and evolutionary forces shaping microbial diversity in the human intestine. Cell. 2006;124:837-48.

2. Cho I, Blaser MJ. The human microbiome: at the interface of health and disease. Nat Rev Genet. 2012;13:260-70.

3. Baumler AJ, Sperandio V. Interactions between the microbiota and pathogenic bacteria in the gut. Nature. 2016;535:85-93.

4. Honda K, Littman DR. The microbiome in infectious disease and inflammation. Annu Rev Immunol. 2012;30:759-95.

5. Brown RL, Clarke TB. The regulation of host defences to infection by the microbiota. Immunology. 2017;150:1-6.

6. Clarke TB. Microbial programming of systemic innate immunity and resistance to infection. PLoS Pathog. 2014;10:e1004506. 
7. Belkaid Y, Hand TW. Role of the microbiota in immunity and inflammation. Cell. 2014;157:121-41.

8. Mathis D, Benoist C. The influence of the microbiota on type-1 diabetes: on the threshold of a leap forward in our understanding. Immunol Rev. 2012;245:239-49.

9. Kriegel MA, Sefik E, Hill JA, Wu HJ, Benoist C, Mathis D. Naturally transmitted segmented filamentous bacteria segregate with diabetes protection in nonobese diabetic mice. Proc Natl Acad Sci USA. 2011;108:11548-53.

10. Van Praet JT, Donovan E, Vanassche I, Drennan MB, Windels F, Dendooven A, et al. Commensal microbiota influence systemic autoimmune responses. EMBO J. 2015;34:466-74.

11. Caballero S, Kim S, Carter RA, Leiner IM, Susac B, Miller L, et al. Cooperating commensals restore colonization resistance to vancomycin-resistant Enterococcus faecium. Cell Host Microbe. 2017;21:592-602. e4

12. Thiemann S, Smit N, Roy U, Lesker TR, Galvez EJC, Helmecke $\mathrm{J}$, et al. Enhancement of IFNgamma production by distinct commensals Ameliorates Salmonella-induced disease. Cell Host Microbe. 2017;21:682-94. e5

13. Brown RL, Sequeira RP, Clarke TB. The microbiota protects against respiratory infection via GM-CSF signaling. Nat Commun. 2017;8:1512.

14. Clarke TB, Davis KM, Lysenko ES, Zhou AY, Yu Y, Weiser JN. Recognition of peptidoglycan from the microbiota by Nod1 enhances systemic innate immunity. Nat Med. 2010;16:228-31.

15. Deshmukh HS, Liu Y, Menkiti OR, Mei J, Dai N, O'Leary CE, et al. The microbiota regulates neutrophil homeostasis and host resistance to Escherichia coli $\mathrm{K} 1$ sepsis in neonatal mice. Nat Med. 2014;20:524-30.

16. Foster SL, Hargreaves DC, Medzhitov R. Gene-specific control of inflammation by TLR-induced chromatin modifications. Nature. 2007;447:972-8.

17. Sequeira RP, McDonald JAK, Marchesi JR, Clarke TB. Commensal Bacteroidetes protect against Klebsiella pneumoniae colonization and transmission through IL-36 signalling. Nat Microbiol. 2020;5:304-13.

18. Dominguez-Bello MG, Costello EK, Contreras M, Magris M, Hidalgo G, Fierer N, et al. Delivery mode shapes the acquisition and structure of the initial microbiota across multiple body habitats in newborns. Proc Natl Acad Sci USA. 2010;107:11971-5.

19. Dominguez-Bello MG, Blaser MJ, Ley RE, Knight R. Development of the human gastrointestinal microbiota and insights from high-throughput sequencing. Gastroenterology. 2011;140:1713-9.

20. Dethlefsen L, Eckburg PB, Bik EM, Relman DA. Assembly of the human intestinal microbiota. Trends Ecol Evol. 2006;21:517-23.

21. Rakoff-Nahoum S, Kong Y, Kleinstein SH, Subramanian S, Ahern PP, Gordon JI, et al. Analysis of gene-environment interactions in postnatal development of the mammalian intestine. Proc Natl Acad Sci USA. 2015;112:1929-36.

22. Palmer C, Bik EM, DiGiulio DB, Relman DA, Brown PO. Development of the human infant intestinal microbiota. PLoS Biol. 2007;5:e177.

23. Mueller NT, Bakacs E, Combellick J, Grigoryan Z, DominguezBello MG. The infant microbiome development: mom matters. Trends Mol Med. 2015;21:109-17.

24. Ferretti P, Pasolli E, Tett A, Asnicar F, Gorfer V, Fedi S, et al. Mother-to-infant microbial transmission from different body sites shapes the developing infant gut microbiome. Cell Host Microbe. 2018;24:133-45. e5

25. Shao Y, Forster SC, Tsaliki E, Vervier K, Strang A, Simpson N, et al. Stunted microbiota and opportunistic pathogen colonization in caesarean-section birth. Nature. 2019;574:117-21.

26. Galazzo G, van Best N, Bervoets L, Dapaah IO, Savelkoul PH, Hornef MW, et al. Development of the microbiota and associations with birth mode, diet, and atopic disorders in a longitudinal analysis of stool samples, collected from infancy through early childhood. Gastroenterology. 2020;158:1584-96.

27. Stokholm J, Thorsen J, Blaser MJ, Rasmussen MA, Hjelmso M, Shah S, et al. Delivery mode and gut microbial changes correlate with an increased risk of childhood asthma. Sci Transl Med. 2020;12:eaax9929.

28. Rodriguez JM, Murphy K, Stanton C, Ross RP, Kober OI, Juge $\mathrm{N}$, et al. The composition of the gut microbiota throughout life, with an emphasis on early life. Micro Ecol Health Dis. 2015;26:26050.

29. Bergstrom A, Skov TH, Bahl MI, Roager HM, Christensen LB, Ejlerskov KT, et al. Establishment of intestinal microbiota during early life: a longitudinal, explorative study of a large cohort of Danish infants. Appl Environ Microbiol. 2014;80:2889-900.

30. de Steenhuijsen Piters WA, Sanders EA, Bogaert D. The role of the local microbial ecosystem in respiratory health and disease. Philos Trans R Soc Lond B Biol Sci. 2015;370:20140294.

31. Pattaroni C, Watzenboeck ML, Schneidegger S, Kieser S, Wong NC, Bernasconi E, et al. Early-life formation of the microbial and immunological environment of the human airways. Cell Host Microbe. 2018;24:857-65. e4

32. Gensollen T, Iyer SS, Kasper DL, Blumberg RS. How colonization by microbiota in early life shapes the immune system. Science. 2016;352:539-44.

33. Hooper LV, Littman DR, Macpherson AJ. Interactions between the microbiota and the immune system. Science. 2012;336:1268-73.

34. Zhang X, Zhivaki D, Lo-Man R. Unique aspects of the perinatal immune system. Nat Rev Immunol. 2017;17:495-507.

35. Kubinak JL, Round JL. Do antibodies select a healthy microbiota? Nat Rev Immunol. 2016;16:767-74.

36. Pamer EG. Resurrecting the intestinal microbiota to combat antibiotic-resistant pathogens. Science. 2016;352:535-8.

37. Medzhitov R, Janeway C Jr. Innate immunity. N Engl J Med. 2000;343:338-44.

38. Turvey SE, Broide DH. Innate immunity. J Allergy Clin Immunol. 2010;125:S24-32.

39. Nathan C. Points of control in inflammation. Nature. 2002;420: 846-52.

40. Ganal-Vonarburg SC, Hornef MW, Macpherson AJ. Microbialhost molecular exchange and its functional consequences in early mammalian life. Science. 2020;368:604-7.

41. Saenz SA, Taylor BC, Artis D. Welcome to the neighborhood: epithelial cell-derived cytokines license innate and adaptive immune responses at mucosal sites. Immunol Rev. 2008;226: 172-90.

42. Duerr CU, Hornef MW. The mammalian intestinal epithelium as integral player in the establishment and maintenance of hostmicrobial homeostasis. Semin Immunol. 2012;24:25-35.

43. Brandl K, Plitas G, Mihu CN, Ubeda C, Jia T, Fleisher M, et al. Vancomycin-resistant enterococci exploit antibiotic-induced innate immune deficits. Nature. 2008;455:804-7.

44. Donaldson GP, Lee SM, Mazmanian SK. Gut biogeography of the bacterial microbiota. Nat Rev Microbiol. 2016;14:20-32.

45. Chassin C, Kocur M, Pott J, Duerr CU, Gutle D, Lotz M, et al. miR-146a mediates protective innate immune tolerance in the neonate intestine. Cell Host Microbe. 2010;8:358-68.

46. Naik S, Kelly EJ, Meijer L, Pettersson S, Sanderson IR. Absence of Toll-like receptor 4 explains endotoxin hyporesponsiveness in human intestinal epithelium. J Pediatr Gastroenterol Nutr. 2001;32:449-53.

47. Kaci G, Lakhdari O, Dore J, Ehrlich SD, Renault P, Blottiere $\mathrm{HM}$, et al. Inhibition of the NF-kappaB pathway in human intestinal epithelial cells by commensal Streptococcus salivarius. Appl Environ Microbiol. 2011;77:4681-4.

48. Lin PW, Myers LE, Ray L, Song SC, Nasr TR, Berardinelli AJ, et al. Lactobacillus rhamnosus blocks inflammatory signaling 
in vivo via reactive oxygen species generation. Free Radic Biol Med. 2009;47:1205-11.

49. Tien MT, Girardin SE, Regnault B, Le Bourhis L, Dillies MA, Coppee JY, et al. Anti-inflammatory effect of Lactobacillus casei on Shigella-infected human intestinal epithelial cells. J Immunol. 2006;176:1228-37.

50. Karmarkar D, Rock KL. Microbiota signalling through MyD88 is necessary for a systemic neutrophilic inflammatory response. Immunology. 2013;140:483-92.

51. Hergott CB, Roche AM, Tamashiro E, Clarke TB, Bailey AG, Laughlin A, et al. Peptidoglycan from the gut microbiota governs the lifespan of circulating phagocytes at homeostasis. Blood. 2016;127:2460-71.

52. Wynn TA, Chawla A, Pollard JW. Macrophage biology in development, homeostasis and disease. Nature. 2013;496:445-55.

53. Geissmann F, Manz MG, Jung S, Sieweke MH, Merad M, Ley K. Development of monocytes, macrophages, and dendritic cells. Science. 2010;327:656-61.

54. Smith PD, Smythies LE, Shen R, Greenwell-Wild T, Gliozzi M, Wahl SM. Intestinal macrophages and response to microbial encroachment. Mucosal Immunol. 2011;4:31-42.

55. Hussell T, Bell TJ. Alveolar macrophages: plasticity in a tissuespecific context. Nat Rev Immunol. 2014;14:81-93.

56. Bain CC, Bravo-Blas A, Scott CL, Perdiguero EG, Geissmann F, Henri S, et al. Constant replenishment from circulating monocytes maintains the macrophage pool in the intestine of adult mice. Nat Immunol. 2014;15:929-37.

57. Chang PV, Hao L, Offermanns S, Medzhitov R. The microbial metabolite butyrate regulates intestinal macrophage function via histone deacetylase inhibition. Proc Natl Acad Sci USA. 2014;111:2247-52.

58. Schulthess J, Pandey S, Capitani M, Rue-Albrecht KC, Arnold I, Franchini F, et al. The short chain fatty acid butyrate imprints an antimicrobial program in macrophages. Immunity. 2019;50: 432-45. e7

59. Ganal SC, Sanos SL, Kallfass C, Oberle K, Johner C, Kirschning $\mathrm{C}$, et al. Priming of natural killer cells by nonmucosal mononuclear phagocytes requires instructive signals from commensal microbiota. Immunity. 2012;37:171-86.

60. Abt MC, Osborne LC, Monticelli LA, Doering TA, Alenghat T, Sonnenberg GF, et al. Commensal bacteria calibrate the activation threshold of innate antiviral immunity. Immunity. 2012;37: 158-70.

61. Arpaia N, Campbell C, Fan X, Dikiy S, van der Veeken J, deRoos P, et al. Metabolites produced by commensal bacteria promote peripheral regulatory $\mathrm{T}$-cell generation. Nature. 2013;504:451-5.

62. Shen Y, Giardino Torchia ML, Lawson GW, Karp CL, Ashwell JD, Mazmanian SK. Outer membrane vesicles of a human commensal mediate immune regulation and disease protection. Cell Host Microbe. 2012;12:509-20.

63. Ichinohe T, Pang IK, Kumamoto Y, Peaper DR, Ho JH, Murray TS, et al. Microbiota regulates immune defense against respiratory tract influenza A virus infection. Proc Natl Acad Sci USA. 2011;108:5354-9.

64. Ruane D, Chorny A, Lee H, Faith J, Pandey G, Shan M, et al. Microbiota regulate the ability of lung dendritic cells to induce IgA class-switch recombination and generate protective gastrointestinal immune responses. J Exp Med. 2016;213:53-73.

65. Eberl G. Immunity by equilibrium. Nat Rev Immunol. 2016;16: 524-32.

66. Valeri M, Raffatellu M. Cytokines IL-17 and IL-22 in the host response to infection. Pathog Dis. 2016;74:ftw111.

67. Sano T, Huang W, Hall JA, Yang Y, Chen A, Gavzy SJ, et al. An IL-23R/IL-22 circuit regulates epithelial serum amyloid A to promote local effector Th17 responses. Cell. 2015;163:381-93.
68. Shaw MH, Kamada N, Kim YG, Nunez G. Microbiota-induced IL-1beta, but not IL-6, is critical for the development of steady-state TH17 cells in the intestine. J Exp Med. 2012;209:251-8.

69. Ivanov II, Atarashi K, Manel N, Brodie EL, Shima T, Karaoz U, et al. Induction of intestinal Th17 cells by segmented filamentous bacteria. Cell. 2009;139:485-98.

70. Atarashi K, Nishimura J, Shima T, Umesaki Y, Yamamoto M, Onoue $\mathrm{M}$, et al. ATP drives lamina propria $\mathrm{T}(\mathrm{H}) 17$ cell differentiation. Nature. 2008;455:808-12.

71. Lochner M, Peduto L, Cherrier M, Sawa S, Langa F, Varona R, et al. In vivo equilibrium of proinflammatory IL-17+ and regulatory IL-10+ Foxp3 + RORgamma $\mathrm{t}+\mathrm{T}$ cells. J Exp Med. 2008;205:1381-93.

72. Josefowicz SZ, Lu LF, Rudensky AY. Regulatory T cells: mechanisms of differentiation and function. Annu Rev Immunol. 2012;30:531-64.

73. Atarashi $\mathrm{K}$, Tanoue $\mathrm{T}$, Shima $\mathrm{T}$, Imaoka A, Kuwahara $\mathrm{T}$, Momose $\mathrm{Y}$, et al. Induction of colonic regulatory $\mathrm{T}$ cells by indigenous Clostridium species. Science. 2011;331:337-41.

74. Park JH, Eberl G. Type 3 regulatory T cells at the interface of symbiosis. J Microbiol. 2018;56:163-71.

75. Hepworth MR, Monticelli LA, Fung TC, Ziegler CG, Grunberg S, Sinha $\mathrm{R}$, et al. Innate lymphoid cells regulate CD4+ T-cell responses to intestinal commensal bacteria. Nature. 2013;498:113-7.

76. Sawa S, Lochner M, Satoh-Takayama N, Dulauroy S, Berard M, Kleinschek M, et al. RORgammat+ innate lymphoid cells regulate intestinal homeostasis by integrating negative signals from the symbiotic microbiota. Nat Immunol. 2011;12:320-6.

77. Rankin LC, Girard-Madoux MJ, Seillet C, Mielke LA, Kerdiles $\mathrm{Y}$, Fenis A, et al. Complementarity and redundancy of IL-22producing innate lymphoid cells. Nat Immunol. 2016;17:179-86.

78. Satoh-Takayama N, Kato T, Motomura $Y$, Kageyama $T$, Taguchi-Atarashi N, Kinoshita-Daitoku R, et al. Bacteriainduced group 2 innate lymphoid cells in the stomach provide immune protection through induction of $\operatorname{IgA}$. Immunity. 2020;52:635-49. e4

79. Kruglov AA, Grivennikov SI, Kuprash DV, Winsauer C, Prepens S, Seleznik GM, et al. Nonredundant function of soluble LTalpha3 produced by innate lymphoid cells in intestinal homeostasis. Science. 2013;342:1243-6.

80. Mortha A, Chudnovskiy A, Hashimoto D, Bogunovic M, Spencer SP, Belkaid Y, et al. Microbiota-dependent crosstalk between macrophages and ILC3 promotes intestinal homeostasis. Science. 2014;343:1249288.

81. Donskey CJ. The role of the intestinal tract as a reservoir and source for transmission of nosocomial pathogens. Clin Infect Dis. 2004;39:219-26.

82. Kim S, Covington A, Pamer EG. The intestinal microbiota: antibiotics, colonization resistance, and enteric pathogens. Immunol Rev. 2017;279:90-105.

83. Gorrie CL, Mirceta M, Wick RR, Edwards DJ, Thomson NR, Strugnell RA, et al. Gastrointestinal carriage is a major reservoir of Klebsiella pneumoniae infection in intensive care patients. Clin Infect Dis. 2017;65:208-15.

84. Podschun R, Ullmann U. Klebsiella spp. as nosocomial pathogens: epidemiology, taxonomy, typing methods, and pathogenicity factors. Clin Microbiol Rev. 1998;11:589-603.

85. Martin RM, Cao J, Brisse S, Passet V, Wu W, Zhao L. et al. Molecular epidemiology of colonizing and infecting isolates of Klebsiella pneumoniae. mSphere. 2016;1:e00261-16.

86. Bagley ST. Habitat association of Klebsiella species. Infect Control. 1985;6:52-8.

87. Magill SS, Edwards JR, Bamberg W, Beldavs ZG, Dumyati G, Kainer MA, et al. Multistate point-prevalence survey of health care-associated infections. N Engl J Med. 2014;370:1198-208. 
88. Martin RM, Bachman MA. Colonization, infection, and the accessory genome of Klebsiella pneumoniae. Front Cell Infect Microbiol. 2018;8:4.

89. Rodriguez-Villar S, Fife A, Baldwin C, Warne RR. Antibioticresistant hypervirulent Klebsiella pneumoniae causing community- acquired liver abscess: an emerging disease. Oxf Med Case Rep. 2019;2019:omz032.

90. Choby JE, Howard-Anderson J, Weiss DS. Hypervirulent Klebsiella pneumoniae - clinical and molecular perspectives. J Intern Med. 2020;287:283-300.

91. Centers for Disease Control and Prevention. Vital signs: carbapenem-resistant Enterobacteriaceae. MMWR Morb Mortal Wkly Rep. 2013;62:165-70.

92. Salomao MC, Guimaraes T, Duailibi DF, Perondi MBM, Letaif LSH, Montal AC, et al. Carbapenem-resistant Enterobacteriaceae in patients admitted to the emergency department: prevalence, risk factors, and acquisition rate. $\mathrm{J}$ Hosp Infect. 2017;97:241-6.

93. Wiener-Well Y, Rudensky B, Yinnon AM, Kopuit P, Schlesinger $\mathrm{Y}$, Broide $\mathrm{E}$, et al. Carriage rate of carbapenem-resistant Klebsiella pneumoniae in hospitalised patients during a national outbreak. J Hosp Infect. 2010;74:344-9.

94. Mills JP, Talati NJ, Alby K, Han JH. The epidemiology of carbapenem-resistant Klebsiella pneumoniae colonization and infection among long-term acute care hospital residents. Infect Control Hosp Epidemiol. 2016;37:55-60.

95. Liu L, Wang Q, Wu X, Qi H, Das R, Lin H, et al. Vancomycin exposure caused opportunistic pathogens bloom in intestinal microbiome by simulator of the human intestinal microbial ecosystem (SHIME). Environ Pollut. 2020;265:114399.

96. Bilinski J, Grzesiowski P, Sorensen N, Madry K, Muszynski J, Robak K, et al. Fecal microbiota transplantation in patients with blood disorders inhibits gut colonization with antibiotic-resistant bacteria: results of a prospective, single-center study. Clin Infect Dis. 2017;65:364-70.

97. Singh R, de Groot PF, Geerlings SE, Hodiamont CJ, Belzer C, Berge I, et al. Fecal microbiota transplantation against intestinal colonization by extended spectrum beta-lactamase producing Enterobacteriaceae: a proof of principle study. BMC Res Notes. 2018;11:190.

98. Sorbara MT, Dubin K, Littmann ER, Moody TU, Fontana E, Seok R, et al. Inhibiting antibiotic-resistant Enterobacteriaceae by microbiota-mediated intracellular acidification. J Exp Med. 2019;216:84-98.

99. Hansen DS, Mestre F, Alberti S, Hernandez-Alles S, Alvarez D, Domenech-Sanchez A, et al. Klebsiella pneumoniae lipopolysaccharide $\mathrm{O}$ typing: revision of prototype strains and O-group distribution among clinical isolates from different sources and countries. J Clin Microbiol. 1999;37:56-62.

100. Wyres KL, Wick RR, Gorrie C, Jenney A, Follador R, Thomson NR, et al. Identification of Klebsiella capsule synthesis loci from whole genome data. Micro Genom. 2016;2:e000102.

101. Lee IR, Molton JS, Wyres KL, Gorrie C, Wong J, Hoh CH, et al. Differential host susceptibility and bacterial virulence factors driving Klebsiella liver abscess in an ethnically diverse population. Sci Rep. 2016;6:29316.

102. Fung CP, Chang FY, Lee SC, Hu BS, Kuo BI, Liu CY, et al. A global emerging disease of Klebsiella pneumoniae liver abscess: is serotype $\mathrm{K} 1$ an important factor for complicated endophthalmitis? Gut. 2002;50:420-4.

103. Yeh KM, Kurup A, Siu LK, Koh YL, Fung CP, Lin JC, et al. Capsular serotype $\mathrm{K} 1$ or $\mathrm{K} 2$, rather than magA and rmpA, is a major virulence determinant for Klebsiella pneumoniae liver abscess in Singapore and Taiwan. J Clin Microbiol. 2007;45:466-71.

104. Struve C, Forestier C, Krogfelt KA. Application of a novel multiscreening signature-tagged mutagenesis assay for identification of Klebsiella pneumoniae genes essential in colonization and infection. Microbiol (Read). 2003;149:167-76.

105. Boll EJ, Nielsen LN, Krogfelt KA, Struve C. Novel screening assay for in vivo selection of Klebsiella pneumoniae genes promoting gastrointestinal colonisation. BMC Microbiol. 2012;12:201.

106. Jung HJ, Littmann ER, Seok R, Leiner IM, Taur Y, Peled J. et al. Genome-wide screening for enteric colonization factors in carbapenem-resistant ST258 Klebsiella pneumoniae. mBio. 2019;10:e02663-18.

107. Tan YH, Chen Y, Chu WHW, Sham LT, Gan YH. Cell envelope defects of different capsule-null mutants in K1 hypervirulent Klebsiella pneumoniae can affect bacterial pathogenesis. Mol Microbiol. 2020;113:889-905.

108. Struve C, Krogfelt KA. Role of capsule in Klebsiella pneumoniae virulence: lack of correlation between in vitro and in vivo studies. FEMS Microbiol Lett. 2003;218:149-54.

109. Fiore E, Van Tyne D, Gilmore MS. Pathogenicity of enterococci. Microbiol Spectr. 2019;7:GPP3-0053-2018.

110. Van Tyne D, Gilmore MS. Friend turned foe: evolution of enterococcal virulence and antibiotic resistance. Annu Rev Microbiol. 2014;68:337-56.

111. Schloissnig S, Arumugam M, Sunagawa S, Mitreva M, Tap J, Zhu A, et al. Genomic variation landscape of the human gut microbiome. Nature. 2013;493:45-50.

112. Jett BD, Huycke MM, Gilmore MS. Virulence of enterococci. Clin Microbiol Rev. 1994;7:462-78.

113. Shankar N, Baghdayan AS, Gilmore MS. Modulation of virulence within a pathogenicity island in vancomycin-resistant Enterococcus faecalis. Nature. 2002;417:746-50.

114. Paulsen IT, Banerjei L, Myers GS, Nelson KE, Seshadri R, Read $\mathrm{TD}$, et al. Role of mobile DNA in the evolution of vancomycinresistant Enterococcus faecalis. Science. 2003;299:2071-4.

115. Montealegre MC, Singh KV, Murray BE. Gastrointestinal tract colonization dynamics by different Enterococcus faecium clades. J Infect Dis. 2016;213:1914-22.

116. Donskey CJ, Chowdhry TK, Hecker MT, Hoyen CK, Hanrahan JA, Hujer AM, et al. Effect of antibiotic therapy on the density of vancomycin-resistant enterococci in the stool of colonized patients. N Engl J Med. 2000;343:1925-32.

117. Ubeda C, Bucci V, Caballero S, Djukovic A, Toussaint NC, Equinda $\mathrm{M}$, et al. Intestinal microbiota containing Barnesiella species cures vancomycin-resistant Enterococcus faecium colonization. Infect Immun. 2013;81:965-73.

118. Pham TA, Clare S, Goulding D, Arasteh JM, Stares MD, Browne HP, et al. Epithelial IL-22RA1-mediated fucosylation promotes intestinal colonization resistance to an opportunistic pathogen. Cell Host Microbe. 2014;16:504-16.

119. Kim SG, Becattini S, Moody TU, Shliaha PV, Littmann ER, Seok $\mathrm{R}$, et al. Microbiota-derived lantibiotic restores resistance against vancomycin-resistant Enterococcus. Nature. 2019;572:665-9.

120. Kommineni S, Bretl DJ, Lam V, Chakraborty R, Hayward M, Simpson $\mathrm{P}$, et al. Bacteriocin production augments niche competition by enterococci in the mammalian gastrointestinal tract. Nature. 2015;526:719-22.

121. Lessa FC, Mu Y, Bamberg WM, Beldavs ZG, Dumyati GK, Dunn JR, et al. Burden of Clostridium difficile infection in the United States. N Engl J Med. 2015;372:825-34.

122. Leffler DA, Lamont JT. Clostridium difficile infection. N Engl J Med. 2015;372:1539-48.

123. Abt MC, McKenney PT, Pamer EG. Clostridium difficile colitis: pathogenesis and host defence. Nat Rev Microbiol. 2016;14:609-20.

124. Slimings C, Riley TV. Antibiotics and hospital-acquired Clostridium difficile infection: update of systematic review and metaanalysis. J Antimicrob Chemother. 2014;69:881-91. 
125. Brown KA, Khanafer N, Daneman N, Fisman DN. Meta-analysis of antibiotics and the risk of community-associated Clostridium difficile infection. Antimicrob Agents Chemother. 2013;57:2326-32.

126. Zackular JP, Moore JL, Jordan AT, Juttukonda LJ, Noto MJ, Nicholson MR, et al. Dietary zinc alters the microbiota and decreases resistance to Clostridium difficile infection. Nat Med. 2016;22:1330-4.

127. Hryckowian AJ, Van Treuren W, Smits SA, Davis NM, Gardner JO, Bouley DM, et al. Microbiota-accessible carbohydrates suppress Clostridium difficile infection in a murine model. Nat Microbiol. 2018;3:662-9.

128. Schnizlein MK, Vendrov KC, Edwards SJ, Martens EC, Young VB. Dietary xanthan gum alters antibiotic efficacy against the murine gut microbiota and attenuates Clostridioides difficile colonization. mSphere. 2020;5:e00708-19.

129. Battaglioli EJ, Hale VL, Chen J, Jeraldo P, Ruiz-Mojica C, Schmidt BA, et al. Clostridioides difficile uses amino acids associated with gut microbial dysbiosis in a subset of patients with diarrhea. Sci Transl Med. 2018;10:eaam7019.

130. Mefferd CC, Bhute SS, Phan JR, Villarama JV, Do DM, Alarcia $\mathrm{S}$, et al. A high-fat/high-protein, atkins-type diet exacerbates Clostridioides (Clostridium) difficile infection in mice, whereas a high-carbohydrate diet protects. mSystems. 2020;5:e00765-19.

131. Suskind DL, Lee D, Solan P, Wahbeh G, Hayden H, Brittnacher $\mathrm{M}$, et al. Dietary therapy for clostridium difficile colonization: a case series. Anaerobe. 2019;57:1-3.

132. Carter GP, Rood JI, Lyras D. The role of toxin A and toxin B in Clostridium difficile-associated disease: Past and present perspectives. Gut Microbes. 2010;1:58-64.

133. Jarchum I, Liu M, Shi C, Equinda M, Pamer EG. Critical role for MyD88-mediated neutrophil recruitment during Clostridium difficile colitis. Infect Immun. 2012;80:2989-96.

134. Ryan A, Lynch M, Smith SM, Amu S, Nel HJ, McCoy CE, et al. A role for TLR4 in Clostridium difficile infection and the recognition of surface layer proteins. PLoS Pathog. 2011;7:e1002076.

135. Hasegawa M, Yamazaki T, Kamada N, Tawaratsumida K, Kim YG, Nunez G, et al. Nucleotide-binding oligomerization domain 1 mediates recognition of Clostridium difficile and induces neutrophil recruitment and protection against the pathogen. $\mathrm{J}$ Immunol. 2011;186:4872-80.

136. Sadighi Akha AA, McDermott AJ, Theriot CM, Carlson PE Jr., Frank CR, McDonald RA, et al. Interleukin-22 and CD160 play additive roles in the host mucosal response to Clostridium difficile infection in mice. Immunology. 2015;144:587-97.

137. Abt MC, Lewis BB, Caballero S, Xiong H, Carter RA, Susac B, et al. Innate immune defenses mediated by two ILC subsets are critical for protection against acute Clostridium difficile infection. Cell Host Microbe. 2015;18:27-37.

138. Buonomo EL, Cowardin CA, Wilson MG, Saleh MM, Pramoonjago P, Petri WA Jr. Microbiota-regulated IL-25 increases eosinophil number to provide protection during Clostridium difficile infection. Cell Rep. 2016;16:432-43.

139. Zar FA, Bakkanagari SR, Moorthi KM, Davis MB. A comparison of vancomycin and metronidazole for the treatment of Clostridium difficile-associated diarrhea, stratified by disease severity. Clin Infect Dis. 2007;45:302-7.

140. Singh T, Bedi P, Bumrah K, Singh J, Rai M, Seelam S. Updates in treatment of recurrent Clostridium difficile infection. J Clin Med Res. 2019;11:465-71.

141. Kyne L, Kelly CP. Recurrent Clostridium difficile diarrhoea. Gut. 2001;49:152-3.

142. van Nood E, Vrieze A, Nieuwdorp M, Fuentes S, Zoetendal EG, de Vos WM, et al. Duodenal infusion of donor feces for recurrent Clostridium difficile. N Engl J Med. 2013;368:407-15.
143. Hvas CL, Dahl Jorgensen SM, Jorgensen SP, Storgaard M, Lemming L, Hansen MM, et al. Fecal microbiota transplantation is superior to fidaxomicin for treatment of recurrent Clostridium difficile infection. Gastroenterology. 2019;156: 1324-32. e3

144. Sbahi H, Di Palma JA. Faecal microbiota transplantation: applications and limitations in treating gastrointestinal disorders. BMJ Open Gastroenterol. 2016;3:e000087.

145. Ng KM, Ferreyra JA, Higginbottom SK, Lynch JB, Kashyap PC, Gopinath S, et al. Microbiota-liberated host sugars facilitate post-antibiotic expansion of enteric pathogens. Nature. 2013;502:96-9.

146. Nagao-Kitamoto H, Leslie JL, Kitamoto S, Jin C, Thomsson KA, Gillilland MG 3rd, et al. Interleukin-22-mediated host glycosylation prevents Clostridioides difficile infection by modulating the metabolic activity of the gut microbiota. Nat Med. 2020;26:608-17.

147. Jia W, Xie G, Jia W. Bile acid-microbiota crosstalk in gastrointestinal inflammation and carcinogenesis. Nat Rev Gastroenterol Hepatol. 2018;15:111-28.

148. Sorg JA, Sonenshein AL. Inhibiting the initiation of Clostridium difficile spore germination using analogs of chenodeoxycholic acid, a bile acid. J Bacteriol. 2010;192:4983-90.

149. Mullish BH, McDonald JAK, Pechlivanis A, Allegretti JR, Kao D, Barker GF, et al. Microbial bile salt hydrolases mediate the efficacy of faecal microbiota transplant in the treatment of recurrent Clostridioides difficile infection. Gut. 2019;68:1791-800.

150. Buffie CG, Bucci V, Stein RR, McKenney PT, Ling L, Gobourne A, et al. Precision microbiome reconstitution restores bile acid mediated resistance to Clostridium difficile. Nature 2015;517:205-8.

151. Lawley TD, Clare S, Walker AW, Stares MD, Connor TR, Raisen C, et al. Targeted restoration of the intestinal microbiota with a simple, defined bacteriotherapy resolves relapsing Clostridium difficile disease in mice. PLoS Pathog. 2012;8:e1002995.

152. Tvede M, Tinggaard M, Helms M. Rectal bacteriotherapy for recurrent Clostridium difficile-associated diarrhoea: results from a case series of 55 patients in Denmark 2000-2012. Clin Microbiol Infect. 2015;21:48-53.

153. Ubeda C, Taur Y, Jenq RR, Equinda MJ, Son T, Samstein M, et al. Vancomycin-resistant Enterococcus domination of intestinal microbiota is enabled by antibiotic treatment in mice and precedes bloodstream invasion in humans. $\mathrm{J}$ Clin Invest. 2010;120:4332-41.

154. Zmora N, Zilberman-Schapira G, Suez J, Mor U, Dori-Bachash $\mathrm{M}$, Bashiardes S, et al. Personalized gut mucosal colonization resistance to empiric probiotics is associated with unique host and microbiome features. Cell. 2018;174:1388-405. e21

155. O'Toole PW, Marchesi JR, Hill C. Next-generation probiotics: the spectrum from probiotics to live biotherapeutics. Nat Microbiol. 2017;2:17057.

156. Krautkramer KA, Fan J, Backhed F. Gut microbial metabolites as multi-kingdom intermediates. Nat Rev Microbiol. 2021; 19:77-94.

157. Koh A, De Vadder F, Kovatcheva-Datchary P, Backhed F. From dietary fiber to host physiology: short-chain fatty acids as key bacterial metabolites. Cell. 2016;165:1332-45.

158. Rea MC, Alemayehu D, Casey PG, O’Connor PM, Lawlor PG, Walsh M, et al. Bioavailability of the anti-clostridial bacteriocin thuricin CD in gastrointestinal tract. Microbiology (Reading). 2014;160:439-45.

159. Makki K, Deehan EC, Walter J, Backhed F. The impact of dietary fiber on gut microbiota in host health and disease. Cell Host Microbe. 2018;23:705-15. 